\title{
On interval-valued optimization problems with generalized invex functions
}

\author{
Izhar Ahmad ${ }^{1,2^{*}}$, Anurag Jayswal $^{3}$ and Jonaki Banerjee ${ }^{3}$
}

${ }^{\text {"Correspondence: }}$

drizhar@kfupm.edu.sa

'Department of Mathematics and

Statistics, King Fahd University of

Petroleum and Minerals, Dhahran,

31261, Saudi Arabia

2Permanent address: Department of

Mathematics, Aligarh Muslim

University, Aligarh, 202 002, India

Full list of author information is

available at the end of the article

\begin{abstract}
This paper is devoted to study interval-valued optimization problems. Sufficient optimality conditions are established for LU optimal solution concept under generalized $(p, r)-\rho-(\eta, \theta)$-invexity. Weak, strong and strict converse duality theorems for Wolfe and Mond-Weir type duals are derived in order to relate the LU optimal solutions of primal and dual problems.
\end{abstract}

MSC: 90C46; 90C26; 90C30

Keywords: nonlinear programming; interval-valued functions; $(p, r)-\rho-(\eta, \theta)$-invexity; LU-optimal; sufficiency; duality

\section{Introduction}

Many real world decision-making problems need to accomplish many objectives: minimize cost, maximize reliability, minimize deviations from desire levels, minimize risk, etc. In these cases optimization problems have a large number of applications. The main goal of single objective optimization is to find the best solution which corresponds to the minimum or maximum value of a single objective function.

In many research fields and real world problems, the methodology for solving optimization problems has been used. There are three kinds of methodology that are used for solving optimization problems, namely deterministic optimization problem, stochastic optimization problem and interval-valued optimization problem. The optimization problems with interval coefficients are termed an interval-valued optimization problem. In this problem, the coefficient is taken as closed intervals. The solution concept imposed upon the objective function is the main difference between the above said three kinds of problems.

Interval programming methods have been used to tackle specific issues in multiple objective linear programming: some deal with uncertainty in the objective functions, others handle uncertainty both in the objective functions and in the RHS of the constraints and others deal with uncertainty in all the coefficients of the model. Charnes et al. [1] proposed an idea for solving the linear programming problems in which the constraints were assumed as closed intervals. Later, Steuer [2] developed an algorithm to solve the linear programming problem with interval objective functions.

Urli and Nadeau [3] presented a process to solve the multi-objective linear programming problems with interval coefficients. Chanas and Kuchta [4] generalized the solution concepts of the linear programming problem with interval coefficients in the objective function based on preference relations between intervals. By imposing a partial ordering

(c) 2013 Ahmad et al.: licensee Springer. This is an Open Access article distributed under the terms of the Creative Commons Attribution License (http://creativecommons.org/licenses/by/2.0), which permits unrestricted use, distribution, and reproduction in any medium, provided the original work is properly cited. 
on the set of all closed intervals, $\mathrm{Wu}[5]$ introduced a solution concept in optimization problems with interval-valued objective functions.

There are several approaches to model uncertainty in optimization problems such as stochastic optimization and fuzzy optimization. Here we consider an optimization problem with interval-valued objective function. Stancu-Minasian and Tigan [6,7] investigated this kind of optimization problem.

$\mathrm{Wu}$ [8] formulated Karush-Kuhn-Tucker optimality conditions for an interval-valued objective function. Later, $\mathrm{Wu}[9,10]$ formulated a Wolfe-type dual problem related to the interval-valued optimization problem and established duality theorems by using the concept of nondominated solution employed in vector optimization problems. Zhou and Wang [11] established a sufficient optimality condition and discussed mixed-type duality for a class of nonlinear interval-valued optimization problems. Recently, Bhurjee and Panda [12] developed a methodology to study the existence of the solutions of an interval optimization problem. Very recently, Zhang et al. [13] discussed the optimality conditions and duality results for interval-valued optimization problems under generalized preinvexity.

Convexity plays an important role in proving the existence of a solution of a general optimization problem. Hence there is a need to study the convex property of interval optimization problems. One of the most useful generalizations of convexity was introduced by Hanson [14]. For details, readers are advised to see [15]. After the concept of $\rho-(\eta, \theta)$ invex function and $(p, r)$-invex function had been introduced by Zalmai [16] and Antczak [17], respectively, Mandal and Nahak[18] proposed a new concept of $(p, r)-\rho-(\eta, \theta)$ invex function and established some symmetric duality results. Recently, Jayswal et al. [19] derived sufficient optimality conditions and duality theorems for interval-valued optimization problems involving generalized convex functions.

In this paper, we consider an interval-valued optimization problem in which the objective function is an interval-valued function and the constraint functions are realvalued, and derive sufficient optimality conditions and duality theorems under generalized $(p, r)-\rho-(\eta, \theta)$-invexity. The organization of the paper is as follows. In Section 2, we recall some definitions and some basic properties related to interval-valued optimization problems. In Section 3, some sufficient optimality conditions for a class of interval-valued programming problems are discussed. Wolfe and Mond-Weir type duality theorems are obtained in Sections 4 and 5, respectively. Conclusion and future work are proposed in Section 6.

\section{Notation and preliminaries}

Let $I$ be a class of all closed and bounded intervals in $R$. Throughout this paper, when we say that $A$ is a closed interval, we mean that $A$ is also bounded in $R$. If $A$ is a closed interval, we use the notation $A=\left[a^{L}, a^{U}\right]$, where $a^{L}$ and $a^{U}$ mean the lower and upper bounds of $A$, respectively. If $a^{L}=a^{U}=a$, then $A=[a, a]=a$ is a real number. Let $A=\left[a^{L}, a^{U}\right]$, $B=\left[b^{L}, b^{U}\right] \in I$, we define

(i) $A+B=\{a+b: a \in A$ and $b \in B\}=\left[a^{L}+b^{L}, a^{U}+b^{U}\right]$,

(ii) $-A=\{-a: a \in A\}=\left[-a^{U},-a^{L}\right]$.

Therefore we see that $A-B=A+(-B)=\left[a^{L}-b^{U}, a^{U}-b^{L}\right]$. We also have

(i) $k+A=\{k+a: a \in A\}=\left[k+a^{L}, k+a^{U}\right]$, 
(ii) $k A=\{k a: a \in A\}=\left\{\begin{array}{ll}{\left[k a^{L}, k a^{U}\right]} & \text { if } k \geq 0, \\ {\left[k a^{U}, k a^{L}\right]} & \text { if } k<0,\end{array}\right.$ where $k$ is a real number.

Let $R^{n}$ denote an $n$-dimensional Euclidean space. The function $F: R^{n} \rightarrow I$ is called an interval-valued function, i.e., $F(x)=F\left(x_{1}, x_{2}, \ldots, x_{n}\right)$ is a closed interval in $R$ for each $x \in R^{n}$. The interval-valued function $F$ can also be written as $F(x)=\left[F^{L}(x), F^{U}(x)\right]$, where $F^{L}(x)$, $F^{U}(x)$ are real-valued functions defined on $R^{n}$ and satisfy the condition $F^{L}(x) \leq F^{U}(x)$ for each $x \in R^{n}$. We note that $[F(x)]^{L}=F^{L}(x)$ and $[F(x)]^{U}=F^{U}(x)$.

In interval mathematics, an order relation is often used to rank interval numbers and it implies that an interval number is better than another but not that one is larger than another. For $A=\left[a^{L}, a^{U}\right]$ and $B=\left[b^{L}, b^{U}\right]$, we write $A \leq_{L U} B$ if and only if $a^{L} \leq b^{L}$ and $a^{U} \leq b^{U}$. It is easy to see that $\leq_{L U}$ is a partial ordering on $I$. Also, we can write $A<_{L U} B$ if and only if $A \leq_{L U} B$ and $A \neq B$.

Equivalently, $A<_{L U} B$ if and only if

$$
\begin{array}{ll}
a^{L}<b^{L}, & a^{U} \leq b^{U} \quad \text { or } \\
a^{L} \leq b^{L}, & a^{U}<b^{U} \quad \text { or } \\
a^{L}<b^{L}, & a^{U}<b^{U} .
\end{array}
$$

Definition 2.1 [18] Let $f: R^{n} \rightarrow R$ be a differentiable function and let $p, r$ be arbitrary real numbers. If there exist $\eta: R^{n} \times R^{n} \rightarrow R^{n}, \theta: R^{n} \times R^{n} \rightarrow R^{n}$ and $\rho \in R$ such that the relations

$$
\begin{aligned}
& \frac{1}{r}\left(\mathrm{e}^{r(f(x)-f(y))}-1\right)(>) \geq \frac{1}{p} \nabla f(y)\left(\mathrm{e}^{p \eta(x, y)}-1\right)+\rho\|\theta(x, y)\|^{2} \quad \text { for } p \neq 0, r \neq 0, \\
& \frac{1}{r}\left(\mathrm{e}^{r(f(x)-f(y))}-1\right)(>) \geq \nabla f(y) \eta(x, y)+\rho\|\theta(x, y)\|^{2} \quad \text { for } p=0, r \neq 0, \\
& f(x)-f(y)(>) \geq \frac{1}{p} \nabla f(y)\left(\mathrm{e}^{p \eta(x, y)}-1\right)+\rho\|\theta(x, y)\|^{2} \quad \text { for } p \neq 0, r=0, \\
& f(x)-f(y)(>) \geq \nabla f(y) \eta(x, y)+\rho\|\theta(x, y)\|^{2} \quad \text { for } p=0, r=0
\end{aligned}
$$

hold, then $f$ is said to be (strictly) $(p, r)-\rho-(\eta, \theta)$-invex at the point $y$ on $R^{n}$ with respect to $\eta, \theta$.

Definition 2.2 Let $f: R^{n} \rightarrow R$ be a differentiable function and let $p, r$ be arbitrary real numbers. If there exist $\eta: R^{n} \times R^{n} \rightarrow R^{n}, \theta: R^{n} \times R^{n} \rightarrow R^{n}$ and $\rho \in R$ such that the relations

$$
\begin{aligned}
& \frac{1}{p} \nabla f(y)\left(\mathrm{e}^{p \eta(x, y)}-1\right)+\rho\|\theta(x, y)\|^{2} \geq 0 \Rightarrow \frac{1}{r}\left(\mathrm{e}^{r(f(x)-f(y))}-1\right)(>) \geq 0 \quad \text { for } p \neq 0, r \neq 0, \\
& \nabla f(y) \eta(x, y)+\rho\|\theta(x, y)\|^{2} \geq 0 \Rightarrow \frac{1}{r}\left(\mathrm{e}^{r(f(x)-f(y))}-1\right)(>) \geq 0 \quad \text { for } p=0, r \neq 0, \\
& \frac{1}{p} \nabla f(y)\left(\mathrm{e}^{p \eta(x, y)}-1\right)+\rho\|\theta(x, y)\|^{2} \geq 0 \Rightarrow f(x)-f(y)(>) \geq 0 \quad \text { for } p \neq 0, r=0, \\
& \nabla f(y) \eta(x, y)+\rho\|\theta(x, y)\|^{2} \geq 0 \Rightarrow f(x)-f(y)(>) \geq 0 \quad \text { for } p=0, r=0
\end{aligned}
$$

hold, then $f$ is said to be (strictly) $(p, r)-\rho-(\eta, \theta)$-pseudo-invex at the point $y$ on $R^{n}$ with respect to $\eta, \theta$. 
Definition 2.3 Let $f: R^{n} \rightarrow R$ be a differentiable function and let $p, r$ be arbitrary real numbers. If there exist $\eta: R^{n} \times R^{n} \rightarrow R^{n}, \theta: R^{n} \times R^{n} \rightarrow R^{n}$ and $\rho \in R$ such that the relations

$$
\begin{aligned}
& \frac{1}{r}\left(\mathrm{e}^{r(f(x)-f(y))}-1\right) \leq 0 \Rightarrow \frac{1}{p} \nabla f(y)\left(\mathrm{e}^{p \eta(x, y)}-1\right) \leq-\rho\|\theta(x, y)\|^{2} \quad \text { for } p \neq 0, r \neq 0, \\
& \frac{1}{r}\left(\mathrm{e}^{r(f(x)-f(y))}-1\right) \leq 0 \Rightarrow \nabla f(y) \eta(x, y) \leq-\rho\|\theta(x, y)\|^{2} \quad \text { for } p=0, r \neq 0, \\
& f(x)-f(y) \leq 0 \Rightarrow \frac{1}{p} \nabla f(y)\left(\mathrm{e}^{p \eta(x, y)}-1\right) \leq-\rho\|\theta(x, y)\|^{2} \quad \text { for } p \neq 0, r=0, \\
& f(x)-f(y) \leq 0 \Rightarrow \nabla f(y) \eta(x, y) \leq-\rho\|\theta(x, y)\|^{2} \quad \text { for } p=0, r=0
\end{aligned}
$$

hold, then $f$ is said to be $(p, r)-\rho-(\eta, \theta)$-quasi-invex at the point $y$ on $R^{n}$ with respect to $\eta, \theta$.

Remark 2.1 It should be noted that the exponentials appearing on the right-hand sides of inequalities above are understood to be taken componentwise and $\mathbf{1}=(1,1, \ldots, 1) \in R^{n}$.

Remark 2.2 All theorems in this paper will be proved only in the case when $p \neq 0, r \neq 0$ (other cases can be dealt with likewise since the only changes arise in a form of inequality). Moreover, without loss of generality, we shall assume that $r>0, p>0$ (in the case when $r<0, p<0$, the direction of some of the inequalities in the proof of the theorems should be changed to the opposite one).

In this paper, we consider the following primal optimization problem with intervalvalued objective function:

(IVP) $\min F(x)=\left[F^{L}(x), F^{U}(x)\right]$ subject to $g_{j}(x) \leq 0, \quad j=1,2, \ldots, m$,

where $F: R^{n} \rightarrow I$ is an interval-valued function and $g_{j}: R^{n} \rightarrow R$ is a real-valued function. Let $\Omega=\left\{x \in R^{n}: g_{j}(x) \leq 0, j=1,2, \ldots, m\right\}$ be the set of all feasible solutions of (IVP). We also denote by $\operatorname{Obj}(F, \Omega)=\{F(x): x \in \Omega\}$ the set of all objective values of primal problem (IVP).

Definition 2.4 [20] Let $x^{*}$ be a feasible solution of the primal problem (IVP). We say that $x^{*}$ is a LU optimal solution of problem (IVP) if there exists no $x_{0} \in \Omega$ such that $F\left(x_{0}\right)<L U$ $F\left(x^{*}\right)$.

Theorem 2.1 (Karush-Kuhn-Tucker type conditions [9]) Assume that $x$ "is a LU optimal solution of primal problem (IVP) and $F, g_{j}, j=1,2, \ldots, m$, are differentiable at $x^{*}$. Suppose that the constraint function $g_{j}, j=1,2, \ldots, m$, satisfies the suitable Kuhn-Tucker constraint qualification [9] at $x^{*}$. Then there exist multipliers $0<\xi^{L}, \xi^{U} \in R$ and $0 \leq \mu_{j} \in R$, $j=1,2, \ldots, m$, such that

$$
\begin{aligned}
& \xi^{L} \nabla F^{L}\left(x^{*}\right)+\xi^{U} \nabla F^{U}\left(x^{*}\right)+\sum_{j=1}^{m} \mu_{j} \nabla g_{j}\left(x^{*}\right)=0, \\
& \mu_{j} g_{j}\left(x^{*}\right)=0, \quad j=1,2, \ldots, m .
\end{aligned}
$$




\section{Sufficient optimality conditions}

In this section, we shall establish the following sufficient optimality conditions for (IVP).

Theorem 3.1 (Sufficiency) Let $x^{*} \in \Omega$ be a feasible solution of (IVP). Suppose that the objective function $F$ and the constraint function $g_{j}, j=1,2, \ldots, m$, are differentiable at $x^{*}$. Assume that $F^{L}$ and $F^{U}$ are $(p, r)-\rho_{1}-(\eta, \theta)$-invex and $(p, r)-\rho_{2}-(\eta, \theta)$-invex, respectively, with respect to $\eta, \theta$ and $\sum_{j=1}^{m} \mu_{j} g_{j}$ is $(p, r)-\rho_{3}-(\eta, \theta)$-invex with respect to $\eta, \theta$ at $x^{*}$ with $\left(\xi^{L} \rho_{1}+\xi^{U} \rho_{2}+\rho_{3}\right) \geq 0$. If there exist (Lagrange) multipliers $0<\xi^{L}, \xi^{U} \in R$ and $\mu=\left(\mu_{1}, \mu_{2}, \ldots, \mu_{m}\right), 0 \leq \mu_{j} \in R, j=1,2, \ldots, m$, such that $\left(x^{*}, \xi^{L}, \xi^{U}, \mu\right)$ satisfies (1) and (2), then $x^{*}$ is a LU optimal solution to problem (IVP).

Proof Let $x^{*}$ be not a LU optimal solution of (IVP). Then there exists a feasible solution $x_{0} \in \Omega$ such that

$$
F\left(x_{0}\right)<L U F\left(x^{*}\right)
$$

That is,

$$
\left\{\begin{array} { l } 
{ F ^ { L } ( x _ { 0 } ) < F ^ { L } ( x ^ { * } ) , } \\
{ F ^ { U } ( x _ { 0 } ) < F ^ { U } ( x ^ { * } ) , }
\end{array} \text { or } \quad \left\{\begin{array} { l } 
{ F ^ { L } ( x _ { 0 } ) \leq F ^ { L } ( x ^ { * } ) , } \\
{ F ^ { U } ( x _ { 0 } ) < F ^ { U } ( x ^ { * } ) , }
\end{array} \text { or } \quad \left\{\begin{array}{l}
F^{L}\left(x_{0}\right)<F^{L}\left(x^{*}\right), \\
F^{U}\left(x_{0}\right) \leq F^{U}\left(x^{*}\right) .
\end{array}\right.\right.\right.
$$

Since $r>0$, using the property of an exponential function, we obtain

$$
\begin{aligned}
& \left\{\begin{array} { l } 
{ \frac { 1 } { r } [ e ^ { r \{ F ^ { L } ( x _ { 0 } ) - F ^ { L } ( x ^ { * } ) \} } - 1 ] < 0 , } \\
{ \frac { 1 } { r } [ e ^ { r \{ F ^ { U } ( x _ { 0 } ) - F ^ { U } ( x ^ { * } ) \} } - 1 ] < 0 , }
\end{array} \quad \text { or } \quad \left\{\begin{array}{l}
\frac{1}{r}\left[e^{r\left\{F^{L}\left(x_{0}\right)-F^{L}\left(x^{*}\right)\right\}}-1\right] \leq 0, \\
\frac{1}{r}\left[e^{r\left\{F^{U}\left(x_{0}\right)-F^{U}\left(x^{*}\right)\right\}}-1\right]<0,
\end{array}\right.\right. \text { or } \\
& \left\{\begin{array}{l}
\frac{1}{r}\left[e^{r\left\{F^{L}\left(x_{0}\right)-F^{L}\left(x^{*}\right)\right\}}-1\right]<0, \\
\frac{1}{r}\left[e^{r\left\{F^{U}\left(x_{0}\right)-F^{U}\left(x^{*}\right)\right\}}-1\right] \leq 0 .
\end{array}\right.
\end{aligned}
$$

Using the above inequalities and the $(p, r)-\rho_{1}-(\eta, \theta)$-invexity of $F^{L}$ and the $(p, r)-\rho_{2}-$ $(\eta, \theta)$-invexity of $F^{U}$ at $x^{*}$, we get

$$
\begin{aligned}
& \left\{\begin{array}{l}
\frac{1}{p}\left\{\nabla F^{L}\left(x^{*}\right)\right\}\left(e^{p \eta\left(x_{0}, x^{*}\right)}-\mathbf{1}\right)+\rho_{1}\left\|\theta\left(x_{0}, x^{*}\right)\right\|^{2}<0, \\
\frac{1}{p}\left\{\nabla F^{U}\left(x^{*}\right)\right\}\left(e^{p \eta\left(x_{0}, x^{*}\right)}-\mathbf{1}\right)+\rho_{2}\left\|\theta\left(x_{0}, x^{*}\right)\right\|^{2}<0,
\end{array}\right. \\
& \left\{\begin{array}{l}
\frac{1}{p}\left\{\nabla F^{L}\left(x^{*}\right)\right\}\left(e^{p \eta\left(x_{0}, x^{*}\right)}-\mathbf{1}\right)+\rho_{1}\left\|\theta\left(x_{0}, x^{*}\right)\right\|^{2} \leq 0, \quad \text { or } \\
\frac{1}{p}\left\{\nabla F^{U}\left(x^{*}\right)\right\}\left(e^{p \eta\left(x_{0}, x^{*}\right)}-\mathbf{1}\right)+\rho_{2}\left\|\theta\left(x_{0}, x^{*}\right)\right\|^{2}<0,
\end{array}\right. \\
& \left\{\begin{array}{l}
\frac{1}{p}\left\{\nabla F^{L}\left(x^{*}\right)\right\}\left(e^{p \eta\left(x_{0}, x^{*}\right)}-\mathbf{1}\right)+\rho_{1}\left\|\theta\left(x_{0}, x^{*}\right)\right\|^{2}<0, \\
\frac{1}{p}\left\{\nabla F^{U}\left(x^{*}\right)\right\}\left(e^{p \eta\left(x_{0}, x^{*}\right)}-\mathbf{1}\right)+\rho_{2}\left\|\theta\left(x_{0}, x^{*}\right)\right\|^{2} \leq 0 .
\end{array}\right.
\end{aligned}
$$

Since $\xi^{L}>0$ and $\xi^{U}>0$, from the above inequalities, we get

$$
\begin{aligned}
& \frac{1}{p}\left\{\xi^{L} \nabla F^{L}\left(x^{*}\right)+\xi^{U} \nabla F^{U}\left(x^{*}\right)\right\}\left(e^{p \eta\left(x_{0}, x^{*}\right)}-\mathbf{1}\right) \\
& \quad+\xi^{L} \rho_{1}\left\|\theta\left(x_{0}, x^{*}\right)\right\|^{2}+\xi^{U} \rho_{2}\left\|\theta\left(x_{0}, x^{*}\right)\right\|^{2}<0 .
\end{aligned}
$$


On the other hand, from the feasibility of $x_{0}$ to (IVP), we have

$$
g_{j}\left(x_{0}\right) \leq 0, \quad j=1,2, \ldots, m
$$

Since $\mu_{j} \geq 0, j=1,2, \ldots, m$, the above inequality together with (2) yields

$$
\sum_{j=1}^{m} \mu_{j} g_{j}\left(x_{0}\right) \leq \sum_{j=1}^{m} \mu_{j} g_{j}\left(x^{*}\right)
$$

As $r>0$, using the property of an exponential function, we get

$$
\frac{1}{r}\left[e^{r\left\{\sum_{j=1}^{m} \mu_{j} g_{j}\left(x_{0}\right)-\sum_{j=1}^{m} \mu_{j} g_{j}\left(x^{*}\right)\right\}}-1\right] \leq 0,
$$

which together with the assumption that $\sum_{j=1}^{m} \mu_{j} g_{j}$ is $(p, r)-\rho_{3}-(\eta, \theta)$-invex at $x^{*}$ gives

$$
\frac{1}{p} \sum_{j=1}^{m} \mu_{j} \nabla g_{j}\left(x^{*}\right)\left(e^{p \eta\left(x_{0}, x^{*}\right)}-\mathbf{1}\right)+\rho_{3}\left\|\theta\left(x_{0}, x^{*}\right)\right\|^{2} \leq 0 .
$$

On adding (3) and (4), we get

$$
\begin{aligned}
& \frac{1}{p}\left(e^{p \eta\left(x_{0}, x^{*}\right)}-1\right)\left[\xi^{L} \nabla F^{L}\left(x^{\prime \prime}\right)+\xi^{U} \nabla F^{U}\left(x^{\prime \prime}\right)+\sum_{j=1}^{m} \mu_{j} \nabla g_{j}\left(x^{\prime \prime}\right)\right] \\
& +\left(\xi^{L} \rho_{1}+\xi^{U} \rho_{2}+\rho_{3}\right)\left\|\theta\left(x_{0}, x^{\prime \prime}\right)\right\|^{2}<0 .
\end{aligned}
$$

Therefore, from the hypothesis that $\left(\xi^{L} \rho_{1}+\xi^{U} \rho_{2}+\rho_{3}\right) \geq 0$ and the above inequality, we get

$$
\frac{1}{p}\left(e^{p \eta\left(x_{0}, x^{*}\right)}-1\right)\left[\xi^{L} \nabla F^{L}\left(x^{*}\right)+\xi^{U} \nabla F^{U}\left(x^{*}\right)+\sum_{j=1}^{m} \mu_{j} \nabla g_{j}\left(x^{*}\right)\right]<0,
$$

which contradicts (1). Therefore $x^{*}$ is a LU optimal solution of (IVP). This completes the proof.

Theorem 3.2 (Sufficiency) Let $x^{*} \in \Omega$ be a feasible solution of (IVP). Suppose that the objective function $F$ and the constraint function $g_{j}, j=1,2, \ldots, m$, are differentiable at $x^{*}$. Assume that $\left(\xi^{L} F^{L}+\xi^{U} F^{U}\right)$ is $(p, r)-\rho_{1}-(\eta, \theta)$-pseudo-invex with respect to $\eta, \theta$ and $\sum_{j=1}^{m} \mu_{j} g_{j}$ is $(p, r)-\rho_{2}-(\eta, \theta)$-quasi-invex with respect to $\eta, \theta$ at $x^{*}$ with $\left(\rho_{1}+\rho_{2}\right) \geq 0$. If there exist (Lagrange) multipliers $0<\xi^{L}, \xi^{U} \in R$ and $\mu=\left(\mu_{1}, \mu_{2}, \ldots, \mu_{m}\right), 0 \leq \mu_{j} \in R$, $j=1,2, \ldots, m$, such that $\left(x^{*}, \xi^{L}, \xi^{U}, \mu\right)$ satisfies (1) and (2), then $x^{*}$ is a LU optimal solution to problem (IVP).

Proof Let $x^{*}$ be not a LU optimal solution of (IVP). Then there exists a feasible solution $x_{0} \in \Omega$ such that

$$
F\left(x_{0}\right)<_{L U} F\left(x^{\prime \prime}\right) .
$$


That is,

$$
\left\{\begin{array} { l } 
{ F ^ { L } ( x _ { 0 } ) < F ^ { L } ( x ^ { * } ) , } \\
{ F ^ { U } ( x _ { 0 } ) < F ^ { U } ( x ^ { * } ) , }
\end{array} \text { or } \quad \left\{\begin{array} { l } 
{ F ^ { L } ( x _ { 0 } ) \leq F ^ { L } ( x ^ { * } ) , } \\
{ F ^ { U } ( x _ { 0 } ) < F ^ { U } ( x ^ { * } ) , }
\end{array} \text { or } \quad \left\{\begin{array}{l}
F^{L}\left(x_{0}\right)<F^{L}\left(x^{*}\right), \\
F^{U}\left(x_{0}\right) \leq F^{U}\left(x^{*}\right) .
\end{array}\right.\right.\right.
$$

Since $\xi^{L}>0$ and $\xi^{U}>0$, from the above inequalities, we have

$$
\xi^{L} F^{L}\left(x_{0}\right)+\xi^{U} F^{U}\left(x_{0}\right)<\xi^{L} F^{L}\left(x^{*}\right)+\xi^{U} F^{U}\left(x^{*}\right) .
$$

As $r>0$, using the property of an exponential function, we get

$$
\frac{1}{r}\left[e^{r\left\{\left(\xi^{L} F^{L}\left(x_{0}\right)+\xi^{U} F^{U}\left(x_{0}\right)\right)-\left(\xi^{L} F^{L}\left(x^{*}\right)+\xi^{U} F^{U}\left(x^{*}\right)\right)\right\}}-1\right]<0,
$$

which together with the assumption that $\xi^{L} F^{L}+\xi^{U} F^{U}$ is $(p, r)-\rho_{1}-(\eta, \theta)$-pseudo-invex at $x^{*}$ gives

$$
\frac{1}{p}\left\{\xi^{L} \nabla F^{L}\left(x^{*}\right)+\xi^{U} \nabla F^{U}\left(x^{*}\right)\right\}\left(e^{p \eta\left(x_{0}, x^{*}\right)}-\mathbf{1}\right)+\rho_{1}\left\|\theta\left(x_{0}, x^{*}\right)\right\|^{2}<0 .
$$

On the other hand, from the feasibility of $x_{0}$ to (IVP), we have

$$
g_{j}\left(x_{0}\right) \leq 0, \quad j=1,2, \ldots, m \text {. }
$$

Since $\mu_{j} \geq 0, j=1,2, \ldots, m$, the above inequality together with (2) yields

$$
\sum_{j=1}^{m} \mu_{j} g_{j}\left(x_{0}\right) \leq \sum_{j=1}^{m} \mu_{j} g_{j}\left(x^{*}\right)
$$

As $r>0$, using the property of an exponential function, we get

$$
\frac{1}{r}\left[e^{r\left\{\sum_{j=1}^{m} \mu_{j} g_{j}\left(x_{0}\right)-\sum_{j=1}^{m} \mu_{j} g_{j}\left(x^{*}\right)\right\}}-1\right] \leq 0,
$$

which together with the assumption that $\sum_{j=1}^{m} \mu_{j} g_{j}$ is $(p, r)-\rho_{2}-(\eta, \theta)$-quasi-invex at $x^{*}$ gives

$$
\frac{1}{p} \sum_{j=1}^{m} \mu_{j} \nabla g_{j}\left(x^{*}\right)\left(e^{p \eta\left(x_{0}, x^{*}\right)}-\mathbf{1}\right)+\rho_{2}\left\|\theta\left(x_{0}, x^{*}\right)\right\|^{2} \leq 0 .
$$

On adding (5) and (6), we get

$$
\begin{aligned}
& \frac{1}{p}\left(e^{p \eta\left(x_{0}, x^{*}\right)}-\mathbf{1}\right)\left[\xi^{L} \nabla F^{L}\left(x^{*}\right)+\xi^{U} \nabla F^{U}\left(x^{*}\right)+\sum_{j=1}^{m} \mu_{j} \nabla g_{j}\left(x^{*}\right)\right] \\
& \quad+\left(\rho_{1}+\rho_{2}\right)\left\|\theta\left(x_{0}, x^{*}\right)\right\|^{2}<0 .
\end{aligned}
$$

Since $\left(\rho_{1}+\rho_{2}\right) \geq 0$, we have

$$
\frac{1}{p}\left(e^{p \eta\left(x_{0}, x^{*}\right)}-\mathbf{1}\right)\left[\xi^{L} \nabla F^{L}\left(x^{*}\right)+\xi^{U} \nabla F^{U}\left(x^{*}\right)+\sum_{j=1}^{m} \mu_{j} \nabla g_{j}\left(x^{*}\right)\right]<0,
$$


which contradicts (1). Therefore $x^{*}$ is a LU optimal solution of (IVP). This completes the proof.

\section{Wolfe-type duality}

In this section, we consider the following Wolfe-type dual problem:

$$
\begin{aligned}
& \text { (WD) } \quad \max F(y)+\sum_{j=1}^{m} \mu_{j} g_{j}(y) \\
& \text { subject to } \\
& \xi^{L} \nabla F^{L}(y)+\xi^{U} \nabla F^{U}(y)+\sum_{j=1}^{m} \mu_{j} \nabla g_{j}(y)=0, \\
& \xi^{L}>0, \xi^{U}>0, \mu_{j} \geq 0, j=1,2, \ldots, m,
\end{aligned}
$$

where $F(y)+\sum_{j=1}^{m} \mu_{j} g_{j}(y)=\left[F^{L}(y)+\sum_{j=1}^{m} \mu_{j} g_{j}(y), F^{U}(y)+\sum_{j=1}^{m} \mu_{j} g_{j}(y)\right]$ is an interval-valued function.

Definition 4.1 Let $\left(y^{*}, \xi^{* L}, \xi^{*}, \mu^{*}\right)$ be a feasible solution of dual problem (WD). We say that $\left(y^{*}, \xi^{* L}, \xi^{* U}, \mu^{*}\right)$ is a LU optimal solution of dual problem (WD) if there exists no $\left(y, \xi^{* L}, \xi^{* U}, \mu^{*}\right)$ such that $F\left(y^{*}\right)+\sum_{j=1}^{m} \mu_{j}^{*} g_{j}\left(y^{*}\right)<L U F(y)+\sum_{j=1}^{m} \mu_{j}^{* \prime} g_{j}(y)$.

Theorem 4.1 (Weak duality) Let $X_{0}$ be an open subset of $R^{n}$. Let $F$ and $g_{j}, j=1,2, \ldots, m$, be differentiable on $X_{0}$. Suppose that $x$ and $\left(y, \xi^{L}, \xi^{U}, \mu\right)$ are the feasible solutions to (IVP) and (WD), respectively. Further assume that $\xi^{L}>0, \xi^{U}>0$ and $\mu_{j} \geq 0, j=1,2, \ldots, m$, such that $\xi^{L} F^{L}+\xi^{U} F^{U}+\sum_{j=1}^{m} \mu_{j} g_{j}$ is $(p, r)-\rho-(\eta, \theta)$-invex with respect to $\eta, \theta$ at $y$ with $\xi^{L}+\xi^{U}=1$ and $\rho \geq 0$. Then

$$
F(x) \geq_{L U} F(y)+\sum_{j=1}^{m} \mu_{j} g_{j}(y) .
$$

Proof Suppose contrary to the result that

$$
F(x)<_{L U} F(y)+\sum_{j=1}^{m} \mu_{j} g_{j}(y)
$$

That is,

$$
\begin{aligned}
& \left\{\begin{array} { l } 
{ F ^ { L } ( x ) < F ^ { L } ( y ) + \sum _ { j = 1 } ^ { m } \mu _ { j } g _ { j } ( y ) , } \\
{ F ^ { U } ( x ) < F ^ { U } ( y ) + \sum _ { j = 1 } ^ { m } \mu _ { j } g _ { j } ( y ) , }
\end{array} \text { or } \left\{\begin{array}{l}
F^{L}(x) \leq F^{L}(y)+\sum_{j=1}^{m} \mu_{j} g_{j}(y), \\
F^{U}(x)<F^{U}(y)+\sum_{j=1}^{m} \mu_{j} g_{j}(y),
\end{array}\right.\right. \text { or } \\
& \left\{\begin{array}{l}
F^{L}(x)<F^{L}(y)+\sum_{j=1}^{m} \mu_{j} g_{j}(y), \\
F^{U}(x) \leq F^{U}(y)+\sum_{j=1}^{m} \mu_{j} g_{j}(y) .
\end{array}\right.
\end{aligned}
$$

Since $\xi^{L}>0, \xi^{U}>0$ and $\xi^{L}+\xi^{U}=1$, the above inequalities together with the feasibility of $x$ to (IVP) become

$$
\xi^{L} F^{L}(x)+\xi^{U} F^{U}(x)+\sum_{j=1}^{m} \mu_{j} g_{j}(x)<\xi^{L} F^{L}(y)+\xi^{U} F^{U}(y)+\sum_{j=1}^{m} \mu_{j} g_{j}(y) .
$$


From the assumption that $\xi^{L} F^{L}+\xi^{U} F^{U}+\sum_{j=1}^{m} \mu_{j} g_{j}$ is $(p, r)-\rho-(\eta, \theta)$-invex at $y$, we have

$$
\begin{aligned}
& \frac{1}{r}\left[e^{r\left(\xi\left(\xi^{L} F^{L}(x)+\xi^{U} F^{U}(x)+\sum_{j=1}^{m} \mu_{j} g_{j}(x)\right)-\left(\xi^{L} F^{L}(y)+\xi^{U} F^{U}(y)+\sum_{j=1}^{m} \mu_{j} g_{j}(y)\right)\right\}}-1\right] \\
& \quad \geq \frac{1}{p}\left[\xi^{L} \nabla F^{L}(y)+\xi^{U} \nabla F^{U}(y)+\sum_{j=1}^{m} \mu_{j} \nabla g_{j}(y)\right]\left(e^{p \eta(x, y)}-1\right)+\rho\|\theta(x, y)\|^{2} .
\end{aligned}
$$

The above inequality together with (7) and $\rho \geq 0$ yields

$$
\frac{1}{r}\left[e^{r\left\{\left(\xi^{L} F^{L}(x)+\xi^{U} F^{U}(x)+\sum_{j=1}^{m} \mu_{j} g_{j}(x)\right)-\left(\xi^{L} F^{L}(y)+\xi^{U} F^{U}(y)+\sum_{j=1}^{m} \mu_{j} g_{j}(y)\right)\right\}}-1\right] \geq 0 .
$$

Since $r>0$, using the property of an exponential function, we get

$$
\xi^{L} F^{L}(x)+\xi^{U} F^{U}(x)+\sum_{j=1}^{m} \mu_{j} g_{j}(x) \geq \xi^{L} F^{L}(y)+\xi^{U} F^{U}(y)+\sum_{j=1}^{m} \mu_{j} g_{j}(y)
$$

which contradicts the inequality (9). This completes the proof.

Theorem 4.2 (Strong duality) Let $x^{*}$ be a LU optimal solution to (IVP) at which KuhnTucker constraints qualification are satisfied. Then there exist $\xi^{* L}>0, \xi^{* U}>0$ and $\mu^{*} \geq 0$ such that $\left(x^{*}, \xi^{* L}, \xi^{* U}, \mu^{*}\right)$ is feasible for (WD) and the two objectives have the same value. Further, if the hypothesis of weak duality Theorem 4.1 holds for all feasible solutions $\left(y^{*}, \xi^{* L}, \xi^{* U}, \mu^{*}\right)$, then $\left(x^{*}, \xi^{* L}, \xi^{* U}, \mu^{*}\right)$ is a LU optimal solution to (WD).

Proof Since $x^{*}$ is a LU optimal solution to (IVP) and the Kuhn-Tucker constraints qualification are satisfied at $x^{*}$, then by Theorem 2.1 , there exist multipliers $\xi^{* L}>0, \xi^{* U}>0$ and $\mu_{j}^{*} \geq 0, j=1,2, \ldots, m$, such that

$$
\begin{aligned}
& \xi^{* L} \nabla F^{L}\left(x^{*}\right)+\xi^{*} \nabla F^{U}\left(x^{*}\right)+\sum_{j=1}^{m} \mu_{j}^{*} \nabla g_{j}\left(x^{*}\right)=0, \\
& \mu_{j}^{*} g_{j}\left(x^{*}\right)=0,
\end{aligned}
$$

which yields that $\left(x^{*}, \xi^{* L}, \xi^{* U}, \mu^{*}\right)$ is a feasible solution for (WD) and corresponding objective values are the same. Further, if $\left(x^{*}, \xi^{* L}, \xi^{* U}, \mu^{* *}\right)$ is not a LU optimal solution of (WD), then there exists a feasible solution $\left(y^{*}, \xi^{* L}, \xi^{*}, \mu^{*}\right)$ for (WD) such that

$$
F\left(x^{*}\right)<_{L U} F\left(y^{*}\right)+\sum_{j=1}^{m} \mu_{j}^{*} g_{j}\left(y^{*}\right)
$$

which contradicts weak duality Theorem 4.1. Hence $\left(x^{*}, \xi^{* L}, \xi^{* U}, \mu^{*}\right)$ is a LU optimal solution to (WD).

Theorem 4.3 (Strict converse duality) Let $X_{0}$ be an open subset of $R^{n}$. Let $F$ and $g_{j}$, $j=1,2, \ldots, m$, be differentiable on $X_{0}$. Suppose that $x^{*}$ and $\left(y^{*}, \xi^{* L}, \xi^{* U}, \mu^{*}\right)$ are the feasible solutions to (IVP) and (WD), respectively. Assume that $\xi^{* L} F^{L}+\xi^{* U} F^{U}+\sum_{j=1}^{m} \mu_{j}^{*} g_{j}$ is 
strictly $(p, r)-\rho-(\eta, \theta)$-invex with respect to $\eta, \theta$ at $y^{*}$ with $\rho \geq 0$ and

$$
\xi^{* L} F^{L}\left(x^{*}\right)+\xi^{* U} F^{U}\left(x^{*}\right)+\sum_{j=1}^{m} \mu_{j}^{*} g_{j}\left(x^{*}\right) \leq \xi^{* L} F^{L}\left(y^{*}\right)+\xi^{* U} F^{U}\left(y^{*}\right)+\sum_{j=1}^{m} \mu_{j}^{*} g_{j}\left(y^{*}\right) .
$$

Then $x^{*}=y^{*}$.

Proof Now we assume that $x^{*} \neq y^{*}$ and exhibit a contradiction. From the assumption that $\xi^{* L} F^{L}+\xi^{* U} F^{U}+\sum_{j=1}^{m} \mu_{j}^{*} g_{j}$ is strictly $(p, r)-\rho-(\eta, \theta)$-invex at $y^{*}$, we have

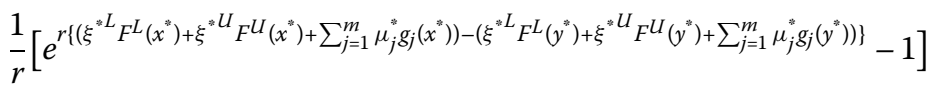

$$
\begin{aligned}
& >\frac{1}{p}\left[\xi^{* L} \nabla F^{L}\left(y^{*}\right)+\xi^{* U} \nabla F^{U}\left(y^{*}\right)+\sum_{j=1}^{m} \mu_{j}^{*} \nabla g_{j}\left(y^{*}\right)\right]\left(e^{p \eta\left(x^{*}, y^{*}\right)}-1\right)+\rho\left\|\theta\left(x^{*}, y^{*}\right)\right\|^{2} .
\end{aligned}
$$

From the feasibility of $\left(y^{*}, \xi^{* L}, \xi^{*}, \mu^{*}\right)$ to (WD), the above inequality together with the hypothesis $\rho \geq 0$ yields

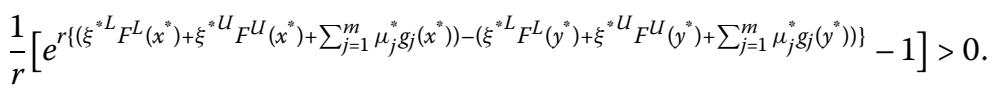

Since $r>0$, using the property of an exponential function, we get

$$
\xi^{* L} F^{L}\left(x^{*}\right)+\xi^{* U} F^{U}\left(x^{*}\right)+\sum_{j=1}^{m} \mu_{j}^{*} g_{j}\left(x^{*}\right)>\xi^{* L} F^{L}\left(y^{*}\right)+\xi^{* U} F^{U}\left(y^{*}\right)+\sum_{j=1}^{m} \mu_{j}^{*} g_{j}\left(y^{*}\right),
$$

which contradicts (10). This completes the proof.

\section{Mond-Weir type duality}

In this section, we consider the following Mond-Weir type dual problem for (IVP):

$$
\begin{array}{ll}
(\mathrm{MWD}) & \max F(y)=\left[F^{L}(y), F^{U}(y)\right] \\
& \text { subject to } \\
& \xi^{L} \nabla F^{L}(y)+\xi^{U} \nabla F^{U}(y)+\sum_{j=1}^{m} \mu_{j} \nabla g_{j}(y)=0, \\
& \mu_{j} g_{j}(y) \geq 0, j=1,2, \ldots, m, \\
& \xi^{L}>0, \xi^{U}>0, \mu_{j} \geq 0, j=1,2, \ldots, m .
\end{array}
$$

Definition 5.1 Let $\left(y^{*}, \xi^{* L}, \xi^{* U}, \mu^{*}\right)$ be a feasible solution of dual problem (MWD). We say that $\left(y^{*}, \xi^{* L}, \xi^{* U}, \mu^{*}\right)$ is a LU optimal solution of dual problem (MWD) if there exists no $\left(y, \xi^{* L}, \xi^{* U}, \mu^{*}\right)$ such that $F\left(y^{*}\right)<_{L U} F(y)$.

Theorem 5.1 (Weak duality) Let $X_{0}$ be an open subset of $R^{n}$. Let $F$ and $g_{j}, j=1,2, \ldots, m$, be differentiable on $X_{0}$. Suppose that $x$ and $\left(y, \xi^{L}, \xi^{U}, \mu\right)$ are the feasible solutions to (IVP) and (MWD), respectively. Further assume that there exist $\xi^{L}>0$ and $\xi^{U}>0$ and $\mu_{j} \geq 0, j=$ 
$1,2, \ldots, m$, such that $\left(\xi^{L} F^{L}+\xi^{U} F^{U}\right)$ is $(p, r)-\rho_{1}-(\eta, \theta)$-pseudo-invex with respect to $\eta, \theta$ and $\sum_{j=1}^{m} \mu_{j} g_{j}$ is $(p, r)-\rho_{2}-(\eta, \theta)$-quasi-invex with respect to $\eta, \theta$ at $y$ with $\left(\rho_{1}+\rho_{2}\right) \geq 0$. Then

$$
F(x) \geq_{L U} F(y)
$$

Proof Suppose contrary to the result that

$$
F(x)<_{L U} F(y) \text {. }
$$

That is,

$$
\left\{\begin{array} { l } 
{ F ^ { L } ( x ) < F ^ { L } ( y ) , } \\
{ F ^ { U } ( x ) < F ^ { U } ( y ) , }
\end{array} \text { or } \quad \left\{\begin{array} { l } 
{ F ^ { L } ( x ) \leq F ^ { L } ( y ) , } \\
{ F ^ { U } ( x ) < F ^ { U } ( y ) , }
\end{array} \text { or } \quad \left\{\begin{array}{l}
F^{L}(x)<F^{L}(y), \\
F^{U}(x) \leq F^{U}(y) .
\end{array}\right.\right.\right.
$$

Since $\xi^{L}>0$ and $\xi^{U}>0$, from the above inequalities, we have

$$
\xi^{L} F^{L}(x)+\xi^{U} F^{U}(x)<\xi^{L} F^{L}(y)+\xi^{U} F^{U}(y) .
$$

On the other hand, since $\mu_{j} \geq 0, j=1,2, \ldots, m$, from the feasibility of $x$ and $\left(y, \xi^{L}, \xi^{U}, \mu\right)$ to (IVP) and (MWD), respectively, we obtain

$$
\sum_{j=1}^{m} \mu_{j} g_{j}(x) \leq \sum_{j=1}^{m} \mu_{j} g_{j}(y)
$$

Since $r>0$, using the property of an exponential function, we get

$$
\frac{1}{r}\left[e^{r\left\{\sum_{j=1}^{m} \mu_{j} g_{j}(x)-\sum_{j=1}^{m} \mu_{j} g_{j}(y)\right\}}-1\right] \leq 0,
$$

which together with the assumption that $\sum_{j=1}^{m} \mu_{j} g_{j}$ is $(p, r)-\rho_{2}-(\eta, \theta)$-quasi-invex at $y$, gives

$$
\frac{1}{p} \sum_{j=1}^{m} \mu_{j} \nabla g_{j}(y)\left(e^{p \eta(x, y)}-\mathbf{1}\right)+\rho_{2}\|\theta(x, y)\|^{2} \leq 0 .
$$

Therefore, from (11) and the hypothesis that $\left(\rho_{1}+\rho_{2}\right) \geq 0$, the above inequality yields

$$
\frac{1}{p}\left(e^{p \eta(x, y)}-\mathbf{1}\right)\left[\xi^{L} \nabla F^{L}(y)+\xi^{U} \nabla F^{U}(y)\right]+\rho_{1}\|\theta(x, y)\|^{2} \geq 0,
$$

which together with the assumption that $\xi^{L} F^{L}+\xi^{U} F^{U}$ is $(p, r)-\rho_{1}-(\eta, \theta)$-pseudo-invex at $y$ gives

$$
\frac{1}{r}\left[e^{\left.r\left(\xi^{L} F^{L}(x)+\xi^{U} F^{U}(x)\right)-\left(\xi^{L} F^{L}(y)+\xi^{U} F^{U}(y)\right)\right\}}-1\right] \geq 0 .
$$

Since $r>0$, using the property of an exponential function, we get

$$
\xi^{L} F^{L}(x)+\xi^{U} F^{U}(x) \geq \xi^{L} F^{L}(y)+\xi^{U} F^{U}(y),
$$

which contradicts (14). This completes the proof. 
Theorem 5.2 (Strong duality) Let $x^{*}$ be a LU optimal solution to (IVP) at which KuhnTucker constraints qualification are satisfied. Then there exist $\xi^{* L}>0, \xi^{* U}>0$ and $\mu^{*} \geq$ 0 such that $\left(x^{*}, \xi^{* L}, \xi^{\prime \prime}, \mu^{\prime \prime}\right)$ is feasible for (MWD) and the two objectives have the same value. Further, if the hypothesis of weak duality Theorem 5.1 holds for all feasible solutions $\left(y^{*}, \xi^{* L}, \xi^{*}, \mu^{*}\right)$, then $\left(x^{*}, \xi^{* L}, \xi^{*}, \mu^{*}\right)$ is a LU optimal solution to (MWD).

Proof Since $x^{*}$ is a LU optimal solution to (IVP) and the Kuhn-Tucker constraints qualification are satisfied at $x^{*}$, then by Theorem 2.1, there exist multipliers $\xi^{* L}>0, \xi^{* U}>0$, $\mu_{j}^{*} \geq 0, j=1,2, \ldots, m$, such that

$$
\begin{aligned}
& \xi^{* L} \nabla F^{L}\left(x^{*}\right)+\xi^{*} U \nabla F^{U}\left(x^{*}\right)+\sum_{j=1}^{m} \mu_{j}^{*} \nabla g_{j}\left(x^{*}\right)=0, \\
& \mu_{j}^{*} g_{j}\left(x^{*}\right)=0,
\end{aligned}
$$

which yields that $\left(x^{*}, \xi^{* L}, \xi^{*}, \mu^{*}\right)$ is a feasible solution for (MWD) and corresponding objective values are same. Further, if $\left(x^{*}, \xi^{n L}, \xi^{\prime \prime}, \mu^{*}\right)$ is not a LU optimal solution of (MWD), then there exists a feasible solution $\left(y^{*}, \xi^{* L}, \xi^{*}, \mu^{*}\right)$ for (MWD) such that

$$
F\left(x^{*}\right)<L U F\left(y^{*}\right)
$$

which contradicts weak duality Theorem 5 .1. Hence $\left(x^{\prime \prime}, \xi^{\prime \prime L}, \xi^{\prime \prime}, \mu^{\prime \prime}\right)$ is a LU optimal solution to (MWD).

Theorem 5.3 (Strict converse duality) Let $X_{0}$ be an open subset of $R^{n}$. Let $F$ and $g_{j}$, $j=1,2, \ldots, m$, be differentiable on $X_{0}$. Suppose that $x^{*}$ and $\left(y^{\prime \prime}, \xi^{\prime \prime}, \xi^{\prime \prime}, \mu^{\prime \prime}\right)$ are the feasible solutions to (IVP) and (MWD), respectively. Assume that $\xi^{* L} F^{L}+\xi^{* U} F^{U}$ is strictly $(p, r)-\rho_{1}-(\eta, \theta)$-pseudo-invex and $\sum_{j=1}^{m} \mu_{j}^{*} g_{j}$ is $(p, r)-\rho_{2}-(\eta, \theta)$-quasi-invex with respect to $\eta, \theta$ at $y^{*}$ with $\left(\rho_{1}+\rho_{2}\right) \geq 0$ and

$$
\xi^{* L} F^{L}\left(x^{*}\right)+\xi^{* U} F^{U}\left(x^{*}\right) \leq \xi^{* L} F^{L}\left(y^{*}\right)+\xi^{* U} F^{U}\left(y^{*}\right) .
$$

Then $x^{*}=y^{*}$.

Proof Now we assume that $x^{*} \neq y^{*}$ and exhibit a contradiction. From the assumption that $\left(y^{*}, \xi^{* L}, \xi^{*}, \mu^{*}\right)$ is a feasible solution for (MWD), we get

$$
\xi^{* L} \nabla F^{L}\left(y^{*}\right)+\xi^{*} \nabla F^{U}\left(y^{*}\right)+\sum_{j=1}^{m} \mu_{j}^{*} \nabla g_{j}\left(y^{*}\right)=0
$$

Since $\mu_{j}^{*} \geq 0, j=1,2, \ldots, m$, from the feasibility of $x^{*}$ and $\left(y^{*}, \xi^{* L}, \xi^{*}, \mu^{*}\right)$ to (IVP) and (MWD), respectively, we obtain

$$
\sum_{j=1}^{m} \mu_{j}^{\prime \prime} g_{j}\left(x^{*}\right) \leq \sum_{j=1}^{m} \mu_{j}^{*} g_{j}\left(y^{*}\right) .
$$


As $r>0$, using the property of an exponential function, we get

$$
\frac{1}{r}\left[e^{r\left\{\sum_{j=1}^{m} \mu_{j}^{*} g_{j}\left(x^{*}\right)-\sum_{j=1}^{m} \mu_{j}^{*} g_{j}\left(y^{*}\right)\right\}}-1\right] \leq 0,
$$

which together with the assumption that $\sum_{j=1}^{m} \mu_{j}^{\prime \prime} g_{j}$ is $(p, r)-\rho_{2}-(\eta, \theta)$-quasi-invex at $y^{*}$ gives

$$
\frac{1}{p} \sum_{j=1}^{m} \mu_{j}^{*} \nabla g_{j}\left(y^{*}\right)\left(e^{p \eta\left(x^{*}, y^{*}\right)}-\mathbf{1}\right)+\rho_{2}\left\|\theta\left(x^{*}, y^{*}\right)\right\|^{2} \leq 0 .
$$

Therefore, from (16) and the hypothesis that $\left(\rho_{1}+\rho_{2}\right) \geq 0$, the above inequality yields

$$
\frac{1}{p}\left(e^{p \eta\left(x^{*}, y^{*}\right)}-1\right)\left[\xi^{* L} \nabla F^{L}\left(y^{*}\right)+\xi^{*} \nabla F^{U}\left(y^{*}\right)\right]+\rho_{1}\left\|\theta\left(x^{*}, y^{*}\right)\right\|^{2} \geq 0,
$$

which together with the assumption that $\xi^{* L} F^{L}+\xi^{*} F^{U}$ is strictly $(p, r)-\rho_{1}-(\eta, \theta)$-pseudoinvex at $y^{*}$ gives

$$
\frac{1}{r}\left[e^{r\left\{\left(\xi^{n L} F^{L}\left(x^{\prime \prime}\right)+\xi^{* U} F^{U}\left(x^{*}\right)\right)-\left(\xi^{\circ L} F^{L}\left(y^{\prime \prime}\right)+\xi^{*} U F^{U}\left(y^{\prime}\right)\right)\right\}}-1\right]>0 .
$$

Since $r>0$, using the property of an exponential function, we get

$$
\xi^{* L} F^{L}\left(x^{*}\right)+\xi^{*} F^{U}\left(x^{*}\right)>\xi^{* L} F^{L}\left(y^{*}\right)+\xi^{*} U F^{U}\left(y^{*}\right),
$$

which contradicts (15). This completes the proof.

\section{Conclusion}

In this paper, we have derived sufficient optimality conditions for a class of interval-valued optimization problems under generalized invex functions. Weak, strong and strict converse duality theorems are discussed for two types of the dual models. It will be interesting to obtain the optimality and duality theorem for a class of interval-valued programming under generalized invexity assumptions in which the involved functions are non-smooth. Moreover, it will also be interesting to see whether the second-order duality results for a class of interval-valued programming problem hold or not. This will orient the future research of the authors.

\section{Competing interests}

The authors declare that they have no competing interests.

Authors' contributions

All authors carried out the proof. All authors conceived of the study, and participated in its design and coordination. All authors read and approved the final manuscript.

\section{Author details}

'Department of Mathematics and Statistics, King Fahd University of Petroleum and Minerals, Dhahran, 31261, Saudi Arabia. ${ }^{2}$ Permanent address: Department of Mathematics, Aligarh Muslim University, Aligarh, 202 002, India.

${ }^{3}$ Department of Applied Mathematics, Indian School of Mines, Jharkhand, Dhanbad 826 004, India.

\section{Acknowledgements}

Izhar Ahmad thanks the King Fahd University of Petroleum and Minerals, Dhahran-31261, Saudi Arabia for the support under the Internal Project No. IN111037. The authors wish to thank the referees for their several valuable suggestions which have considerably improved the presentation of this article. 


\section{References}

1. Charnes, A, Granot, F, Phillips, F: An algorithm for solving interval linear programming problems. Oper. Res. 25 688-695 (1977)

2. Steuer, RE: Algorithms for linear programming problems with interval objective function coefficients. Math. Oper. Res. 6, 333-348 (1981)

3. Urli, B, Nadeau, R: An interactive method to multiobjective linear programming problems with interval coefficients. INFOR 30, 127-137 (1992)

4. Chanas, S, Kuchta, D: Multiobjective programming in optimization of interval objective functions-a generalized approach. Eur. J. Oper. Res. 94, 594-598 (1996)

5. Wu, H-C: Duality theory for optimization problems with interval-valued objective functions. J. Optim. Theory Appl. $144,615-628(2010)$

6. Stancu-Minasian, IM, Tigan, S: Multiobjective mathematical programming with inexact data. In: Slowiński, R, Teghem, J (eds.) Stochastic versus Fuzzy Approaches to Multiobjective Mathematical Programming under Uncertainty, pp. 395-418. Kluwer Academic, Boston (1990)

7. Stancu-Minasian, IM: Stochastic Programming with Multiple Objective Function. Reidel, Dordrecht (1984)

8. Wu, H-C: The Karush-Kuhn-Tucker optimality conditions in an optimization problem with interval-valued objective function. Eur. J. Oper. Res. 176, 46-59 (2007)

9. $\mathrm{Wu}, \mathrm{H}-\mathrm{C}$ : On interval-valued nonlinear programming problems. J. Math. Anal. Appl. 338, 299-316 (2008)

10. Wu, H-C: Wolfe duality for interval-valued optimization. J. Optim. Theory Appl. 138, 497-509 (2008)

11. Zhou, HC, Wang, YJ: Optimality condition and mixed duality for interval-valued optimization. In: Fuzzy Information and Engineering, Volume 2. Proceedings of the Third International Conference on Fuzzy Information and Engineering (ICFIE 2009). Advances in Intelligent and Soft Computing, vol. 62, pp. 1315-1323. Springer, Berlin (2009)

12. Bhurjee, AK, Panda, G: Efficient solution of interval optimization problem. Math. Methods Oper. Res. 76, 273-288 (2012)

13. Zhang, J, Liu, S, Li, L, Feng, Q: The KKT optimality conditions in a class of generalized convex optimization problems with an interval-valued objective function. Optim. Lett. (2012). doi:10.1007/s11590-012-0601-6

14. Hanson, MA: On sufficiency of the Kuhn-Tucker conditions. J. Math. Anal. Appl. 80, 545-550 (1981)

15. Arana, M, Ruiz, G, Rufián, A (eds.): Optimality Conditions in Vector Optimization. Bentham Science Publishers, Bussum (2010)

16. Zalmai, GJ: Generalized sufficiency criteria in continuous-time programming with application to a class of variational-type inequalities. J. Math. Anal. Appl. 153, 331-355 (1990)

17. Antczak, T: (p,r)-Invex sets and functions. J. Math. Anal. Appl. 263, 355-379 (2001)

18. Mandal, P, Nahak, C: Symmetric duality with $(p, r)-\rho-(\eta, \theta)$-invexity. Appl. Math. Comput. 217, 8141-8148 (2011)

19. Jayswal, A, Stancu-Minasian, IM, Ahmad, I: On sufficiency and duality for a class of interval-valued programming problems. Appl. Math. Comput. 218, 4119-4127 (2011)

20. Sun, Y, Wang, L: Optimality conditions and duality in nondifferentiable interval-valued programming. J. Ind. Manag. Optim. 9, 131-142 (2013)

doi:10.1186/1029-242X-2013-313

Cite this article as: Ahmad et al.: On interval-valued optimization problems with generalized invex functions. Journal of Inequalities and Applications 2013 2013:313.

\section{Submit your manuscript to a SpringerOpen ${ }^{\circ}$ journal and benefit from:}

- Convenient online submission

Rigorous peer review

- Immediate publication on acceptance

- Open access: articles freely available online

- High visibility within the field

- Retaining the copyright to your article 